\title{
Metascintillators: New results for TOFPET applications
}

\author{
P. Lecoq, Fellow, IEEE, G. Konstantinou, R. Latella, L. Moliner, J. Nuyts, L. Zhang, J. Barrio, \\ Member, IEEE, J.M. Benlloch, A.J. Gonzalez, Member, IEEE
}

Abstract- We report on the progress on a $1^{\text {st }}$ generation of realistic size metascintillators for time-of-flight PET. These heterostructures combine dense LYSO or BGO plates, interleaved with fast scintillator layers producing a bunch of prompt photons from the energy leakage of the recoil photoelectric electron. From a Geant 4 simulation of the energy sharing distribution between the dense and the fast scintillator on 42 LYSO-based and 42 BGObased configurations, a detailed study of the timing performance has been performed on a selection of the most promising 12 LYSObased and 14 BGO-based metascintillators.

A Monte Carlo simulation was first performed to extrapolate from direct measurements of the performance of the metascintillator components, the Detector Time Resolution (DTR) and sensitivity on the basis of the simulated amount of energy leakage to the fast scintillator. An analytic algorithm was then applied to determine an equivalent CTR from the random association of the DTR distributions from two metapixels in coincidence. This equivalent CTR is calculated in order to obtain the same variance in the reconstructed image as the combination of the DTR distributions of 2 metapixels. Preliminary results confirm that with these simple, still non-optimized configurations an equivalent CTR of 150ps for BGO-based and 140ps for LYSObased metapixels of realistic size can be obtained.

Index Terms-

Scintillators, metascintillator, PET, Time-of-Flight, heterostructure

\section{INTRODUCTION}

Besides Positron Emission Tomography (PET) provides the best molecular sensitivity and quantitative accuracy among all the other medical imaging modalities, there is a strong motivation for pushing even further the sensitivity, to allow reducing the radiation exposure to the patients and clinicians, reducing the scan time, opening the way to follow-up on slow

This paragraph of the first footnote will contain the date on which you submitted your paper for review. It will also contain support information, including sponsor and financial support acknowledgment. For example, "This work was supported in part by the U.S. Department of Commerce under Grant BS123456."

Paul Lecoq is with Multiwave Metacrystal SA, Geneva, Switzerland, the Instituto de Instrumentación para Imagen Molecular, Centro Mixto CSICUniversitat Politècnica de València, 46022 Valencia, Spain, and CERN dynamic processes with long lifetime isotopes, as well as addressing new medical challenges, such as tracking a small number of cells.

Two routes are extensively explored to reach this ambitious objective. The first one is based on increasing the geometrical acceptance of the PET scanner by extending its axial field of view. This is the Total Body PET approach, initiated by the group at UC Davis [1], already producing impressive results [2].

The second route exploits improving the time-of-flight (TOF) performance leading to an increase in the signal-to-noise ratio (SNR) of the reconstructed image and to a gain in the PET effective sensitivity [3]. The accuracy of TOF is bound to the whole detector chain, namely scintillator, photosensor and readout electronics. One possible approach to overcome the timing resolution limits of standard scintillators commonly used in PET scanners, such as BGO, LSO, LYSO, LGSO, etc, is based on the metascintillator concept proposed in $[4,5]$, a deeptech approach, benefiting from recent important progresses in a number of disruptive technologies to combine and optimize several functionalities in the same scintillator heterostructure, as shown in Fig. 1.

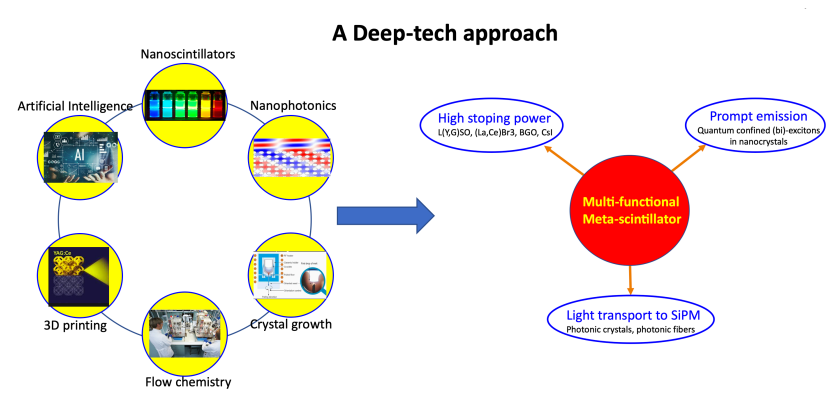

Fig. 1: The Metascintillator concept

The operation principle of metascintillators is based on the possibility to sample in a fast scintillator layer, the recoil electron produced by the photoelectric conversion of the

(European Organization for Nuclear Research), 1211 Geneva, Switzerland (email: paul.lecoq@cern.ch)

G. Konstantinou, R. Latella and L. Zhang are with Multiwave Metacrystal SA, Geneva, Switzerland

J. Barrio, A.J. Gonzalez, L. Moliner, J.M. Benlloch are with the Instituto de Instrumentación para Imagen Molecular, Centro Mixto CSIC-Universitat Politècnica de València 46022 Valencia, Spain

J. Nuyts is with Nuclear Medicine \& Molecular Imaging, MIRC, KU Leuven, Belgium 
incoming gamma ray in a dense host scintillator. The range of the recoil electron is typically 400 to 500 microns in crystals commonly used in PET scanners, such as BGO and LYSO. The spatial frequency of the scintillator heterostructure needs therefore to be designed and implemented at this level [6].

This paper will present the results of a first generation of metascintillators in the context of PET applications. A discussion on the methodology to best exploit the rich information given by the metascintillators will be provided. Finally, perspectives for a second and a third generation of metascintillators will be presented.

\section{MATERIALS AND METHODS}

\section{A. Metascintillator configurations and testing procedure}

A first generation of metascintillators has been produced and tested, based on simple one-dimensional (1D) configuration of BGO (from EPIC, China) or LYSO (from EPIC, SIPAT, China and CPI, USA) plates, acting as the dense host material, and EJ232 and EJ232Q plastic scintillators (from Eljen Technology, USA) or BaF2 (from SICCAS, China) plates, acting as the prompt photon emitters. Our first tests correspond to metascintillators in pixel shaped topologies, what we call metapixels. The plates have dimensions of $3 \times 15 \mathrm{~mm}^{2}$ or $3 \times 20 \mathrm{~mm}^{2}$ and thickness ranging from $50 \mu \mathrm{m}$ to $300 \mu \mathrm{m}$. Metapixels with external dimension of $3 \times 3 \times 15 \mathrm{~mm}^{3}$ and $3 \times 3 \times 20 \mathrm{~mm}^{3}$ have been produced by interleaving plates of both types of scintillators in a parallel geometry (plates parallel to the direction of the incoming $\gamma$ ray), without individually wrapping or special surface treatment of the plates or any attempt yet to improve the light transport and collection, in particular of the fast scintillator. Only the whole metapixel is wrapped with 2 layers of teflon. Perpendicular geometries of larger dimensions $\left(30 \times 30 \times 15 \mathrm{~mm}^{3}\right)$ with two-sided readout have also been considered (see Fig. 2). The drawback of such a geometry is to introduce dead space on 2 sides of the metascintillator block. However, it allows an easy depth of interaction (DOI) determination, and does not require more SiPM coverage area than the pixel geometry with single readout, if the plates have a lateral dimension at least twice the depth of the metascintillator block.

All together, we have studied 84 different metascintillator configurations with this simple 1D integration scheme and different plate thicknesses:

$\begin{array}{ll}\text { - } & 12 \mathrm{BGO} / \mathrm{EJ} 232 \text { parallel } \\ \text { - } & 9 \mathrm{BGO} / \mathrm{BaF} 2 \text { parallel } \\ \text { - } & 12 \mathrm{BGO} / \mathrm{EJ} 232 \text { perpendicular } \\ \text { - } & 9 \mathrm{BGO} / \mathrm{BaF} 2 \text { perpendicular } \\ \text { - } & 12 \mathrm{LYSO} / \mathrm{EJ} 232 \text { parallel } \\ \text { - } & 9 \mathrm{LYSO} / \mathrm{BaF} 2 \text { parallel } \\ - & 12 \mathrm{LYSO} / \mathrm{EJ} 232 \text { perpendicular } \\ \text { - } & 9 \mathrm{LYSO} / \mathrm{BaF} 2 \text { perpendicular }\end{array}$

In order to evaluate their performance, we have measured the coincidence time resolution (CTR) of the separate components

\section{D integration}
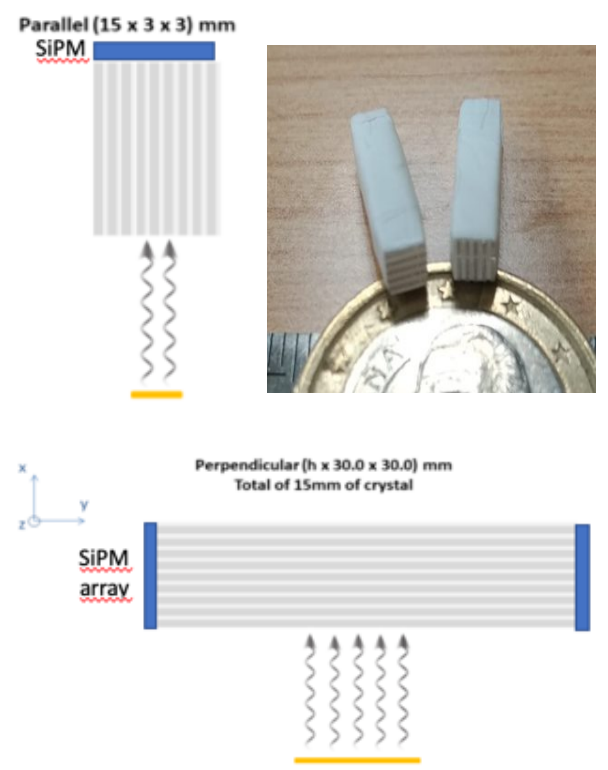

Fig. 2: 1D metascintillator integration schemes in parallel and perpendicular geometries. Picture of a BGO/EJ232 and a LYSO/EJ232 metapixels $3 \times 3 \times 15 \mathrm{~mm}^{3}$

on a test bench. This bench is using a two-channel readout for the energy and timing measurement, with a balloon transformer in the timing channel and an $8 \mathrm{GHz}$ oscilloscope to have enough bandwidth and sensitivity to the prompt emitted photons by the fast scintillator components. More details about this bench are given in [7]. However, the settings have been made in such a way as to obtain CTR values for our reference pixels compatible to the values obtained at a system level. Indeed, we found a CTR of 200ps FWHM for $3 \times 3 \times 20 \mathrm{~mm}^{3}$ bulk LYSO pixels (similar to the value obtained by the Biograph Vision with the same crystal dimensions), $400 \mathrm{ps}$ for $3 \times 3 \times 15 \mathrm{~mm}^{3}$ bulk BGO pixels and 100 ps for $3 \times 3 \times 15 \mathrm{~mm}^{3}$ bulk BaF2 pixels. Our estimate for the EJ232 plastic is also 100ps, based on previous measurements but with different dimensions. In such conditions, we also measure a CTR of 110ps for two reference LYSO:Ce, Ca crystals $3 \times 3 \times 5 \mathrm{~mm}^{3}$. Notice that in our test bench, we have made use of low power consumption electronics, differently from other labs. This has impacted our results, not easily allowing to currently overcome the 100ps barrier [8]. However, the fact that we get on our bench the same CTR of 200 ps for two $3 \times 3 \times 20 \mathrm{~mm}^{3}$ bulk LYSO pixels in coincidence as obtained at a system level in the Biograph Vision, let us believe that our results are representative of the metascintillator performance to be expected in a PET scanner.

For the measurements, the metascintillators using EJ232 plastic scintillator were coupled using a silicon grease to $3 \times 3 \mathrm{~mm}^{2}$ NUV SiPM from FBK with a photodetection efficiency (PDE) of 50\%PDE@370nm (EJ232 emission wavelength), whereas the BGO or LYSO-based metascintillators using $\mathrm{BaF} 2$ were coupled using glycerin to $3 \times 3 \mathrm{~mm}^{2}$ VUV FBK with a PDE of $20 \%$ PDE at $200 \mathrm{~nm}$. 


\section{B. Simulations}

After a screening of the 84 configurations, on which a detailed Geant 4 simulation has been performed of the energy sharing distribution between the dense host and the fast scintillator, the 26 most promising (12 LYSO-based and 14 BGO-based) have been selected to be assembled and tested. Indeed, we are here in a different context as for standard TOFPET systems, as, due to the stochastic nature of the energy sharing between the 2 metascintillator components, not all the coincidence events carry the same timing information. We are therefore dealing with a sum of a large number of Gaussian kernels, which is not Gaussian. For each configuration, we obtain 3 categories of events, each one characterized by a distribution of detector time resolution (DTR), obtained from the measured DTR of the separate components, weighted by the squared root of the energy fraction in the corresponding component:

- Slow events, where all the $511 \mathrm{keV}$ energy is released in the dense scintillator (BGO or LYSO)

- Fast events where all the $511 \mathrm{keV}$ energy is released in the fast scintillator

- Shared events with a variable amount of energy leakage from the dense to the fast scintillator.

These 3 categories of events for each pixel (slow, fast and shared), are leading to 6 timing bands for two metapixels in coincidence, namely slow-slow, fast-fast, shared-shared, slowfast, slow-shared and fast-shared events. We can then determine an equivalent CTR, leading to a reconstructed image having the same variance as the image produced by the CTR distribution of the metascintillators (Fig. 3). This procedure has been detailed and mathematically justified in the following reference [9]. It is interesting to notice that a sizable fraction of events (54.4\% in this case) have a CTR better than the equivalent CTR.

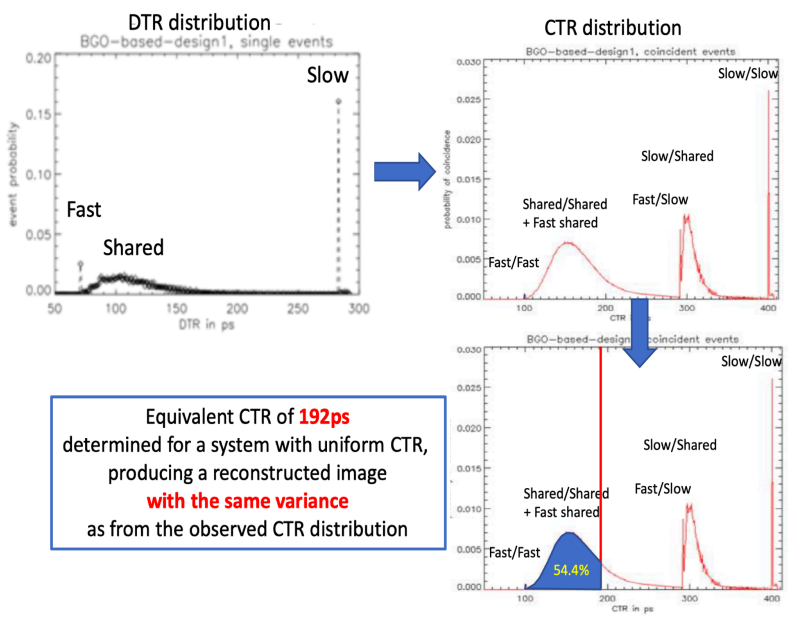

Fig.3: Determination of the equivalent CTR of $2 \mathrm{BGO} / \mathrm{BaF} 2$ metascintillators in coincidence from the DTR distribution in each of them. For this configuration $54.4 \%$ of the events have a CTR better than the equivalent CTR of 192ps

Metapixels corresponding to the most promising configurations have been assembled in order to compare this data driven simulation by direct measurements (Fig 4.).

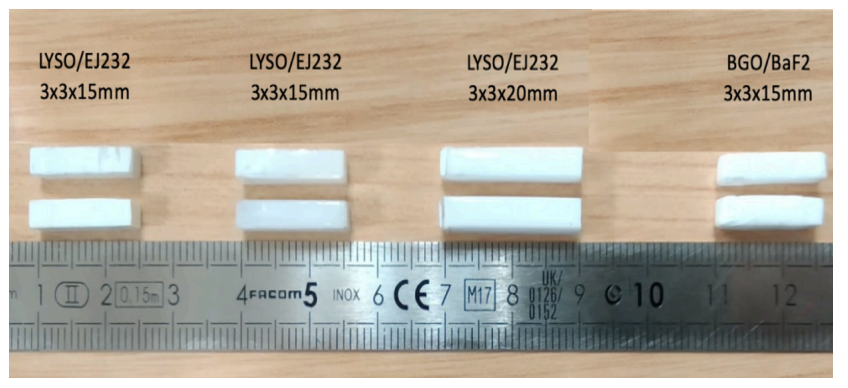

Fig. 4: Picture of a few assembled metapixels wrapped with 2 layers of Teflon tape

Our objective for this first generation of metascintillators is to reach 200ps CTR at a system level for BGO-based configurations, i.e. a similar resolution as the one obtained by the Siemens Biograph Vision PET scanner, but with the 3 times cheaper BGO than LYSO, and 100ps CTR resolution at a system level for LYSO-based configurations.

\section{RESULTS}

\section{A. BGO-based metascintillators}

A bulk $3 \times 3 \times 15 \mathrm{~mm}^{3}$ BGO pixel is taken as a reference in terms of detection sensitivity for $511 \mathrm{keV} \gamma$-rays and CTR, measured on our bench at a value of 400ps.

Fig. 5 shows the energy sharing distribution for a large number of $\mathrm{BGO} / \mathrm{EJ} 232$ (top) and $\mathrm{BGO} / \mathrm{BaF} 2$ (bottom) parallel configurations.
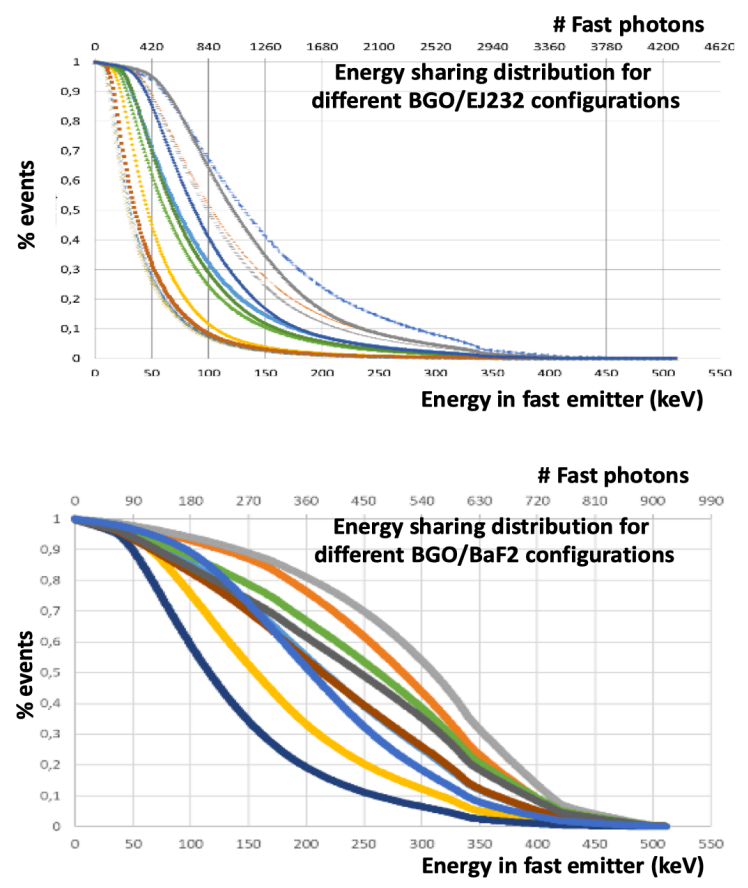

Fig. 5: Energy sharing distribution for several BGO/EJ232 (top) and $\mathrm{BGO} / \mathrm{BaF} 2$ (bottom) configurations. The energy deposited in the fast scintillator is indicated in the bottom horizontal scale as well as the corresponding number of emitted prompt photons (top horizontal scale) 
For these parallel pixel configurations, the combination of $\mathrm{BGO}$ with less dense materials such as $\mathrm{EJ} 232$ or $\mathrm{BaF} 2$ reduces the $511 \mathrm{keV} \gamma$-rays detection efficiency, which must be compensated by an extra length. We have therefore calculated the length of the corresponding metapixels, which would lead to the same $\gamma$-rays detection efficiency as the $3 \times 3 \times 15 \mathrm{~mm}^{3}$ bulk BGO pixel. Fig. 6 shows the equivalent CTR of the most realistic $6 \mathrm{BGO} / \mathrm{BaF} 2$ and $5 \mathrm{BGO} / \mathrm{EJ} 232$ metapixels derived from the data driven simulations described in section IIB, as a function of the required length for having the same detection efficiency as the $3 \times 3 \times 15 \mathrm{~mm}^{3}$ bulk BGO pixel. The comparison with a direct measurement of two of these metapixels shows the excellent match with our data driven simulations. Details of the measurement and data analysis procedure are given in [10].

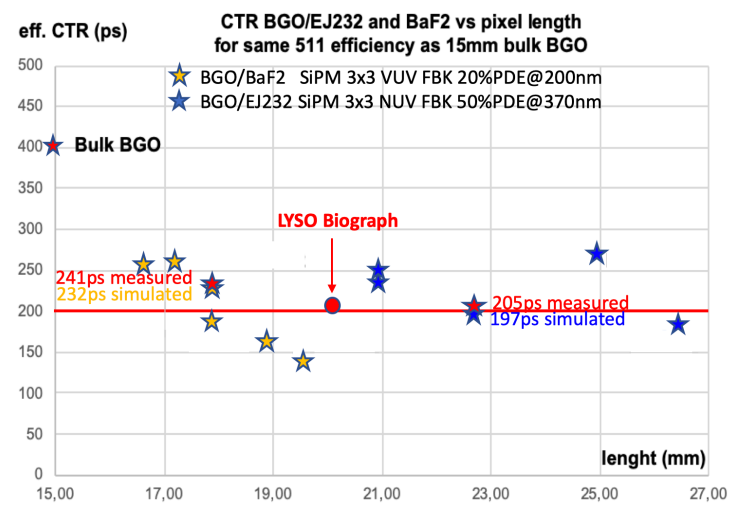

Fig. 6: Equivalent $\mathrm{CTR}$ of different $\mathrm{BGO} / \mathrm{BaF} 2$ and $\mathrm{BGO} / \mathrm{EJ} 232$ parallel configurations metapixels as a function of their required length to have the same detection efficiency as a $3 \times 3 \times 15 \mathrm{~mm}^{3}$ bulk BGO pixel

These results confirm that our strategic target to reach the same 200ps equivalent CTR resolution as obtained with LYSO in the Biograph Vision can be obtained and even surpassed with the 3 times cheaper BGO than LYSO. As shown in Fig. 3, for each of these configurations, a fraction of the events $(20 \%$ to $55 \%$ on this plot) have an even better CTR than the value plotted here.

However, while an even shorter $\mathrm{BGO} / \mathrm{BaF} 2$ metapixel length can be used to reach the same detection efficiency, and better CTR than the $20 \mathrm{~mm}$ bulk LYSO of the Biograph Vision, the low density of plastic imposes the need for a longer metapixel to reach the same detection efficiency, at least for these simple parallel configurations. The situation is of course different for the perpendicular configurations, where as many plates as needed can be stacked to reach a given detection efficiency. CTR values of 209ps and 186ps have been obtained for two perpendicular configurations with EJ232 and BaF2 respectively.

Moreover, this type of arrangement allows the 3D reconstruction of the $\gamma$ interaction point by the identification of the BGO plate where the interaction took place (DOI) and through light sharing on the lateral SiPM array for the $\mathrm{x}$ and $\mathrm{y}$ coordinates.

\section{B. LYSO-based metascintillators}

We have followed the same procedure for LYSO-based configurations with EJ232 and BaF2 for the fast scintillator. Here the reference crystals are $3 \times 3 \times 20 \mathrm{~mm}^{3}$ bulk LYSO pixels, for which we have measured a CTR of 200ps FWHM.

Fig. 7 shows the energy sharing distribution for a large number of LYSO/EJ232 (top) and LYSO/BaF2 (bottom) parallel configurations.

Fig. 8 shows the equivalent CTR of the most realistic 4 LYSO/BaF2 and $5 \mathrm{LYSO} / \mathrm{EJ} 232$ metapixels derived from the data driven simulations as a function of the required length for having the same detection efficiency as a $3 \times 3 \times 15 \mathrm{~mm}^{3}$ bulk BGO pixel.
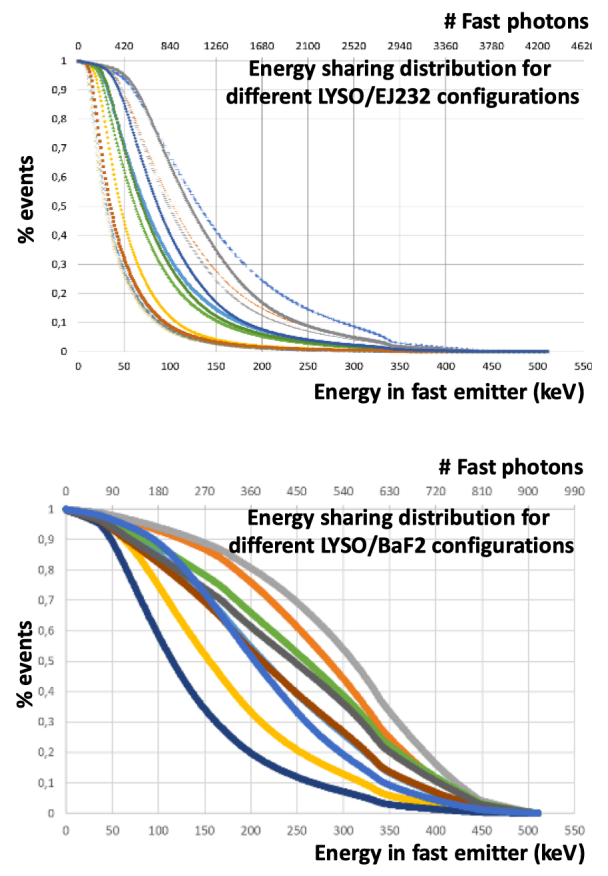

Fig. 7: Energy sharing distribution for several LYSO/EJ232 (top) and LYSO/BaF2 (bottom) configurations. The energy deposited in the fast scintillator is indicated in the bottom horizontal scale as well as the corresponding number of emitted prompt photons (top)

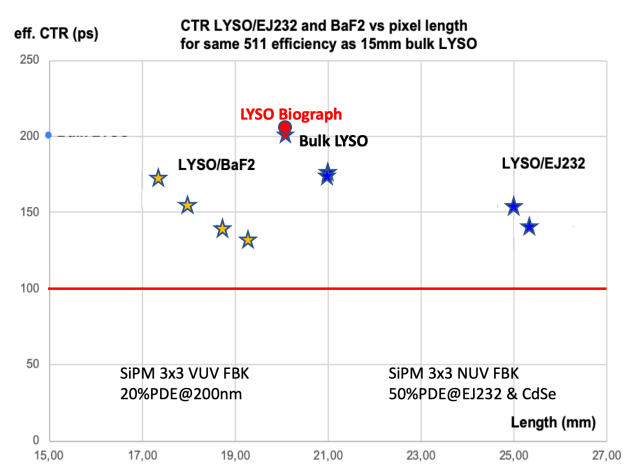

Fig. 8: Equivalent CTR of different LYSO/BaF2 and LYSO/EJ232 parallel configurations metapixels as a function of their required length to have the same detection efficiency as a $3 \times 3 \times 15 \mathrm{~mm}^{3}$ bulk BGO pixel 
Perpendicular configurations give equivalent CTR results ranging between $138 \mathrm{ps}$ and $155 \mathrm{ps}$.

Here we see that all the configurations provide a significant gain as compared to bulk LYSO, but we do not reach our objective of 100ps with plastic or BaF2 when using the VUV SiPM from FBK with only 20\% PDE in the UV. However, our ongoing effort to improve the fast UV light collection efficiency, should allow reaching this limit. Indeed, a simulation with CdSe nanoplatelets embedded in polystyrene with $60 \%$ weight and emitting at $540 \mathrm{~nm}$, where the SiPM quantum efficiency is about $40 \%$, shows that an equivalent CTR of 107 ps can be obtained.

\section{DISCUSSION AND PERSPECTIVES}

\section{A. On the first generation of metascintillators}

The very encouraging results presented in section III have been obtained with easily available commercial components (BGO, LYSO, EJ232 and BaF2) without any attempt to improve their performance in terms of timing resolution. There are indeed two important points, which can be further optimized in the context of metascintillators.

The first one is related to the rate of prompt photons produced by the fast scintillator in the leading edge of the scintillation pulse. In this spirit we are in the process of comparing the EJ232 plastic scintillator with a quenched version of it [11], providing a higher rate of prompt photons in spite of a lower light yield, as shown on Fig. 9.

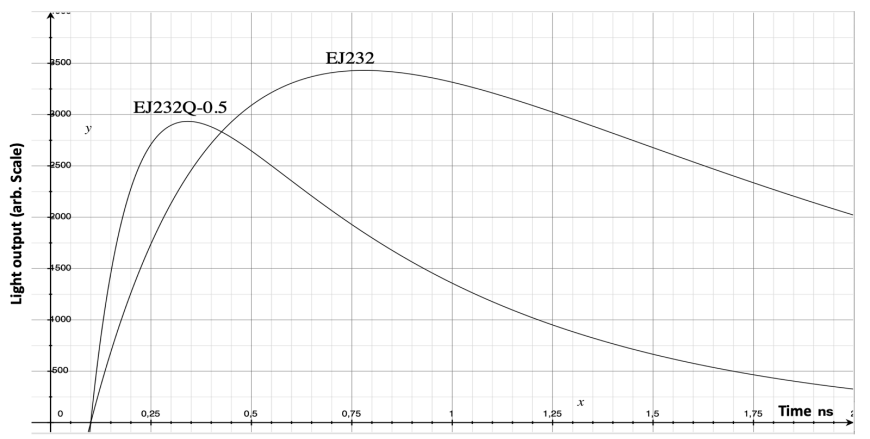

Fig. 9: Pulse shape of EJ232 and EJ232Q-0.5 plastic scintillators (from Eljen technologies data sheets)

Another important consideration for the design of metascintillators is to reach a reasonable balance between the number of fast photons, used to time tag the event with the required precision, and the number of standard scintillation photons allowing a good estimate of the energy. If the latter is comparatively too high, the prompt photon contribution will be more difficult to detect. We estimate a reasonable ratio to be at the level of $10 \%$ for $10 \%$ energy leakage in the fast scintillator. It means that the dense, slow scintillator and the fast scintillator components, should have ideally the same light yield. While this is the case for the $\mathrm{BGO} / \mathrm{EJ} 232$ configuration, it is not for the $\mathrm{BGO} / \mathrm{BaF} 2$, because of the relatively low light yield of the fast $\mathrm{BaF} 2$ component $(1800 \mathrm{ph} / \mathrm{MeV})$, but also because of the additional slow component of $\mathrm{BaF} 2$, which comes in addition to the slow BGO emission. We are therefore preparing tests with yttrium doped BaF2, with a strongly suppressed slow component at $600 \mathrm{~ns}$, while keeping unchanged the fast $800 \mathrm{ps}$ one. [12].

Finally, we are exploring a new, recently patented semimonolithic configuration allowing an independent optimization of the TOF resolution and the 3D information of the $\gamma$-ray interaction point. The principle is to stack on a SiPM array parallel semi-monolithic plates of the dense host scintillator (BGO or LYSO for instance) covering a full row of SiPMs, allowing by light sharing the 3D reconstruction of the $\gamma$-ray interaction point, as explained in [13]. These plates will be interleaved with pixel plates of the fast scintillator, each one coupled one-to-one to the SiPMs. This design will allow maximizing the ratio prompt/slow photons at the level of each SiPM for an optimal time tag determination, while providing a precise DOI information of the $\gamma$-ray interaction point (Fig. 10).

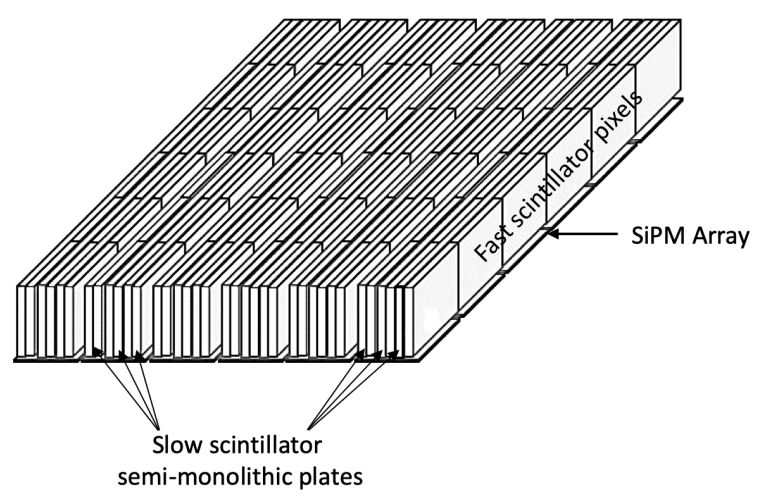

Fig. 10: Arrangement of a semi-monolithic metascintillator block

A specific effort is also ongoing to introduce artificial intelligence (AI) techniques for a fast and reliable direct evaluation of the energy sharing on an event-to-event basis.

\section{B. Towards a second and a third generation of metascintillators}

Capitalizing on the promising results obtained with the first generation of metascintillators, our team in now engaged in the development of a second generation based on the same or similar components, but introducing this time several features to optimize the light transport and light extraction efficiency, while minimizing the photons transit time spread. An attempt will also be made to use $\mathrm{CdSe}, \mathrm{ZnO}$ and perovskite nanoscintillators as the prompt photon emitter, following our preliminary studies on such materials [14]. The objective is to reach an equivalent CTR of $100 \mathrm{ps}$ for $\mathrm{BGO} / \mathrm{BaF} 2$-based and 50ps for LYSO-based configurations.

We are also considering for a longer term a third generation of metascintillators with more exotic nanophotonic features, 2D and even 3D integration schemes and an ultimate objective of 10ps equivalent CTR. 


\section{CONCLUSION}

Metamaterials are a technology enabler. They are designed from conventional materials to engineer bespoke characteristics and functionality. The structure/function relationships of light offer infinite possibilities to make the unimaginable possible and to open the way to groundbreaking applications, such as quantum sensing and multifunctional and even intelligent sensors, which are likely to introduce a paradigm shift in the way to design ionization radiation detectors, among other applications.

In this context, the metascintillator concept is a real game changer, as it allows not only to break the natural barriers of standard bulk scintillators, but it also offers the possibility to engineer the scintillator with a large flexibility of designs, to satisfy on demand different requirements in terms of detection efficiency, energy, position and timing resolution.

\section{ACKNOWLEDGMENT}

This wok has been initiated in the frame of the first author's ERC Advanced Grant TICAL \#338953 and the related Prof-ofConcept project ULTIMA \# 680552, both funded by the European Commission. It has been in part supported by the European Research Council (ERC) under the European Union's Horizon 2020 research and innovation programme (Grant Agreement \#695536) It is pursued through a public-private partnership between the company Multiwave Metacrystal SA, based in Geneva (Switzerland) and the Instituto de Instrumentación para Imagen Molecular, Centro Mixto CSICUniversitat Politècnica de València, 46022 Valencia, Spain. Their support is greatly acknowledged.

\section{REFERENCES}

[1] S.R. Cherry et al., "Total-body imaging: Transforming the role of positron emission tomography", Sci. Transl. Med. 9, eaaf6169 (2017) 15 March 2017.

[2] B.A. Spencer et al., "Performance evaluation of the uEXPLORER TotalBody PET/CT Scanner Based on NEMA NU 2-2018 with Additional Tests to Characterize PET Scanners with Long Axial Field of View", Journal of Nuclear Medicine, June 2021, 62 (6), 861-870.

[3] M Conti and B Bendriem, "The new opportunities for high time resolution clinical TOF PET," Clin and Transl Imaging, vol. 7, no. 2, pp. 139-147, 2019. doi: 10.1007/s40336-019-00316-5.

[4] P. Lecoq, "Metamaterials for novel X- or g-ray detector designs", 2008 IEEE Nuclear Science Symposium Conference Record, N07-1, pp.680684.

[5] P. Lecoq, "Pushing the Limits in Time-of-Flight PET Imaging", IEEE Transactions on Radiation and Plasma Medical Sciences, vol. 1, $n^{\circ} 6, p p$. 473-485, Nov. 2017

[6] G. Konstantinou et al. "Metascintillators for ultra-fast gamma detectors: a review of current state and future perspectives." IEEE Transactions on Radiation and Plasma Medical Sciences (2021)

[7] R. Latella et al., "Test Setup and Data Selection Protocols for the Measurement of Metascintillator CTR", proceedings of the 2021 NSS/MIC conference

[8] S. Gundacker et al., "Measurement of intrinsic rise times for various L(Y)SO and LuAG scintillators with a general study of prompt photons to achieve $10 \mathrm{ps}$ in TOF-PET", Phys. Med. Biol. 61 (2016) 2802-2837.

[9] J. Nuyts et al., "Estimating the relative SNR of individual TOF-PET events for Gaussian and non-Gaussian TOF-kernels",

[10] G. Konstantinou et al., "Metascintillator pulse shape analysis for optimizing energy and timing measurements", proceedings of the 2021 NSS/MIC conference
[11] https://eljentechnology.com/products/plastic-scintillators/ej-232-ej$232 \mathrm{q}$

[12] R.-Y- Zhu et al., "Applications of Very fFst Inorganic cCystal Scintillators in Future HEP Experiments", Proceedings of the TIPP2017 conference, https://doi.org/10.1007/978-981-13-1316-5 13

[13] N. Cucarella et al., "Timing evaluation of a PET detector block based on semi-monolithic LYSO crystals", Medical Physics, early access, https://doi.org/10.1002/mp.15318, 2021.

[14] R.M. Turtos et al, " Towards a metamaterial approach for fast timing in PET: experimental proof-of-concept." Physics in Medicine \& Biology, 64(18), 185018 